\title{
Effect of vortex on mass transport and mixing in microcapillary channels
}

\author{
Xiangsong Feng ${ }^{\mathrm{a}}$, Yukun Ren ${ }^{\mathrm{a}, \mathrm{b}, \mathrm{c}, *}$, Haizhen Sun ${ }^{\mathrm{a}}$, Xiaoming Chen ${ }^{\mathrm{a}}$, Kailiang Zhang ${ }^{\mathrm{a}}$, Yankai Jia $^{\mathrm{a}}$, \\ Likai Hou $^{\mathrm{a}}$, Mingyu Xiao ${ }^{\mathrm{a}}$, Hongyuan Jiang ${ }^{\mathrm{a}, \mathrm{b}, *}$
}

${ }^{a}$ School of Mechatronics Engineering, Harbin Institute of Technology, Harbin 150001, PR China

${ }^{\mathrm{b}}$ State Key Laboratory of Robotics and System, Harbin Institute of Technology, Harbin 150001, PR China

${ }^{\mathrm{c}}$ The State Key Laboratory of Nonlinear Mechanics (LNM), Institute of Mechanics, Chinese Academy of Science, Beijing 100190, PR China

\section{H I G H L I G H T S}

- Joint numerical and experimental research on a novel vortex-based mixer was conducted.

- Different vortex patterns form in the capillary channel as inlet construction varies.

- Not all kinds of vortex flow can intensify mixing process in vortex-based micromixer.

- The vortex pair along the outflow direction shows a higher mixing capability.

\section{A R T I C L E I N F O}

\section{Keywords:}

Micromixing

Vortex-based micromixer

Mixing enhancement

Experimental analysis

Navier-Stokes equations

\begin{abstract}
A B S T R A C T
We present a combined numerical and experimental study of a novel design for a vortex-based mixer by virtue of easy insertion of inlet tubes into a capillary-based square channel. We show that, as the Reynolds number (Re) increases above 72, different patterns of vortices develop by changing the inlet configuration. At relatively higher $R e$, we observe that the disturbance posed by a vortex on the contact surface is not only dependent on its vorticity, but also correlates with the direction of vortex/vortex pair. As a consequence, recognition and application of suitable vortex pattern is of great importance for mixing enhancement. The designed mixer under four inflow confluence conditions, producing single vortex or vortex pair with different directions, shows different mixing performance in the $R e$ range from 20 to 280, from which the optimum design approach capable of generating counter-rotating vortex pair along the outflow direction with higher mixing quality is found. This explains the mass transport and mixing observed in fluid flows with a vortex, which to date has not been studied before.
\end{abstract}

\section{Introduction}

Micromixing and microreaction implemented in a microfabricationbased channel with microliter or even nanoliter volume, have been considered very attractive and effective for different chemical engineering applications [1-5]. However, micromixing in microchannels depends mainly on molecular diffusion, since the fluid is typically stratified under low Reynolds numbers $(R e)$ in microchannels, especially in a straight channel, which will demand a long mixing length for efficient mixing of fluids [6], therefore, to address this issue, many micromixing strategies are put forward [7-9], which are usually divided into two groups: active ones employing external fields, such as acoustic, electric, magnetic fields, et al [10-13], to disturb the fluidic contact surface and speed up the mass transfer rate, and passive ones utilizing complicated channel geometries or multilayer crossing channel structures to shorten the mixing length and enhance the mixing performance [14-17].

One of the convenient and efficient approaches for mixing enhancement is to generate vortex in the micromixing channel. Since suitable vortex pattern is capable of effectively stretching and folding the contact surface between fluid streams, many researchers endeavored to design ingenious structures in microchannels or employ external fields to produce powerful vortex(es) $[6,12,18-22]$. Under low $\operatorname{Re}(\operatorname{Re}=\rho \bar{V} L / \mu, \bar{V}$ is the mean velocity of the fluid, $L$ is the characteristic length) conditions ( $R e<<1$ ), if the fluid is subject to an electric field, the fluid on the driving or floating electrodes will flow as vortices, called AC or induced-charge electro-osmosis vortices, respectively $[19,23,24]$. In another case, vortices can also form in a fluid

\footnotetext{
* Corresponding authors at: School of Mechatronics Engineering, Harbin Institute of Technology, Harbin 150001, PR China.

E-mail addresses: rykhit@hit.edu.cn (Y. Ren), jhy_hit@hit.edu.cn (H. Jiang).
} 
subject to the action of an acoustic or magnetic field [25]. Such vortices are active because external fields are introduced; they have been topical in manipulating microfluids or particles dispersed in fluids [25-27] as they can be easily formed and controlled by adjusting the external fields. However, when the background fluid is faster than the active vortex-induced flow, the disturbance of the vortex on the flow diminishes or even disappears. Hence, these active approaches remain unsuitable for high-throughput mixing situations, especially when $R e$ is higher than 10.

Despite the noteworthy merits of active vortices, including high controllability of both their position and vorticity for flexible manipulation of fluids or particles in fluid, they are usually limited by the selectivity in the proper fluid conductivity, the geometry of the electrodes, and fluid magnetization. Additionally, the impact of active vortices on mass transport and fluid mixing easily deteriorates as the external field-induced flow is slower the background fluid. Fortunately, without the external body fields (apart from gravity), a vortex can still form with the rubbing of one fluid against another or against a wall boundary or a blunt body. To date, although many kinds of vortices are known, whether their direction impacts mass transport still remains elusive.

In particular, M.A. Ansari et al. [20] proposed a novel 3-D T-mixer with non-aligned inputs for vortex generation, which showed higher mixing performance than a typical T-mixer. And Lin et al. [28] reported an eight-inlet mixer to produce a 3-D vortex in circular microchamber above a critical Re. Interestingly, even in a simple T-type micromixer, the fluid will go to different kind of vortex regimes as $R e$ gradually increases [29-34] and the different mixing effectiveness occurs. What's more, significant intensification of mixing could be accomplished in unsteady asymmetric regime [30]. By changing the confluence angle, channel width/height ratio, et al, the flow in the T-type microchannel also witnessed different vortex patterns [31,33,34].

In this study, we performed a joint numerical and experimental investigation of a novel design for a vortex-based mixer by means of easy insertion of inlet tubes into a capillary-based square channel. The fluids confluence conditions in the mixing channel can be conveniently varied by bending of one inlet tube or positioning the inlet tubes in different plane of the mixing channel. We find that the formation of different vortex configurations is subject to different inflow confluence and also demonstrate that the disturbance posed by a vortex on the contact surface of fluid streams is not only dependent on its vorticity, but also relates to the direction of vortex/vortex pair, that is, the existence of vortex in the microchannel may not intensify the mixing process, as a consequence, recognition and application of suitable vortex pattern is of great importance for mixing enhancement, just as above-mentioned researchers' works revealed. The designed mixer under four inflow confluence conditions shows different mixing performance in the $R e$ range from 20 to 280 , from which the optimum design approach capable of generating counter-rotating vortex pair with higher mixing quality is found.

\section{Numerical analysis}

\subsection{Geometric model for generation of vortex}

To understand quantitatively the effect of a vortex on the stretching and rotation of the contact surfaces of the material (which, consequently, enhances the mass transport in fluid), we simulate four situations inside a square capillary channel with a different inlet configuration [Fig. 1(a)]. Here, the square capillary $(400 \mu \mathrm{m} \times 400 \mu \mathrm{m})$ serves as the main channel and the other two round channels (inner diameter (I.D.) of $100 \mu \mathrm{m}$, outer diameter (O.D.) of $170 \mu \mathrm{m}$ ) as inlets. The central lines of the inlet tubes are coplanar, so they determine a plane. As Fig. 1(a) shown, the variable $h$ is the distance from the side wall of the square tube to that plane. $\theta$ is the bending angle from the $x$ axis direction to the $-z$-axis direction. In the four configurations, it is
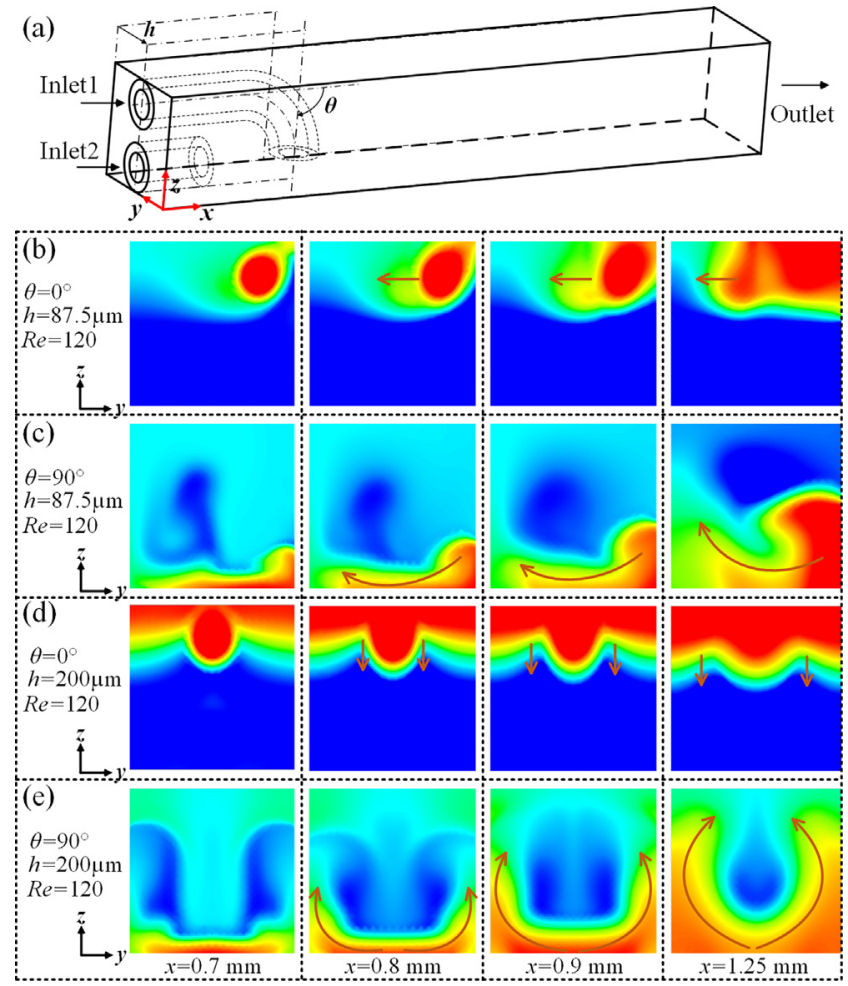

Fig. 1. Model for the generation of a vortex disturbing the surface between fluids. (a) Geometric model with two inlets and one outlet, where the inlet channels are defined by two round capillary tubes with inner diameter of $100 \mu \mathrm{m}$. One is bent at an angle of $\theta$ and the second is straight, apart from the bent tube with a distance of $360 \mu \mathrm{m}$. The inlet channels are inserted into the main channel, which is a square capillary tube of inner side $400 \mu \mathrm{m}$. The plane where these two round capillary tubes are located is at a distance of $h$ from the side wall of the square tube. (b), (c), (d) and (e) are the simulated flow patterns on the transverse sections for $\left(\theta=0^{\circ}, h=87.5 \mu \mathrm{m}\right),\left(\theta=90^{\circ}, h=87.5 \mu \mathrm{m}\right)$, $\left(\theta=0^{\circ}, h=200 \mu \mathrm{m}\right)$ and $\left(\theta=90^{\circ}, h=200 \mu \mathrm{m}\right)$ configurations, respectively, when $R e$ is 120 . The red and blue regions mark the fluid introduced from Inlets 1 and 2, respectively. The green stripes represent layers of contact surface between fluids. (For interpretation of the references to color in this figure legend, the reader is referred to the web version of this article.)

the centers of inlet tube orifices that are distanced by $360 \mu \mathrm{m}$. For the position of the inlet tubes, their outside walls contact the upper and lower walls of the square tube respectively, i.e., each center with distance of $87.5 \mu \mathrm{m}$ to the wall of the square tube. And other dimensions can be calculated based on the above-mentioned ones.

\subsection{Numerical method}

All the numerical simulations were accomplished with the commercial CFD code ANSYS CFX 16.0, to analyze the fluid flow and mixing in the capillary channel. We assume the fluid mechanics inside the channel under different inlet conditions corresponds to an incompressible Newtonian fluid flowing under steady-state conditions. The fluid is a mixture of water and ethyl alcohol (weight ratio 1:1). At temperature of $25^{\circ} \mathrm{C}$, the density and viscosity of this working fluid are $0.861 \times 10^{3} \mathrm{~kg} \cdot \mathrm{m}^{-3}$ and $1.889 \times 10^{-3} \mathrm{~kg} \cdot \mathrm{m}^{-1} \cdot \mathrm{s}^{-1}$, respectively. The two fluids at the two inlets shared the same physical properties and a very small amount of the solute added into the fluids did not influence the mixture density and viscosity. In the simulation, we only simulated the situation where one fluid with the solute and the other fluid without. The mass fraction for the solute in fluids at Inlet 1 and 2 were set 1 and 0 , respectively. We solve the Navier-Stokes and the continuity equations for the velocity and pressure fields through discretization using the control volume finite-difference method [35]. For the 
pressure velocity-coupling algorithm, the coupled multigrid solver is used in the ANSYS CFX software, which can solve the full hydrodynamic system of equations simultaneously. And for the spatial discretization methods, the second order upwind was employed to minimize the numerical diffusion errors resulted from the discretization of the convection terms in the governing equations.

The structured grids of the four geometries (flow domains) were generated with the mesh generator software ANSYS ICEM 16.0. The mesh for each model constructed by hexahedral elements inside the volume with prism-shaped elements near the walls, was employed to provide sufficient resolution for boundary layers near the fluid-solid interfaces [36]. The prism layers can also provide a better control in packing both the corners and the gap between the square channel wall and the inlet tubes with a sufficient number of nodes. In order to obtain mesh independent results from the simulations, a preliminary mesh sensitivity test was implemented to determine the suitable number of elements. Five different mesh systems for case of $\theta=0^{\circ}, h=200 \mu \mathrm{m}$ with the number of cells ranging from $1.39 \times 10^{6}$ to $4.60 \times 10^{6}$ were tested with the mixing efficiency calculated along the mixing channel under the condition of $R e=20$ (see Fig. S1 in the Supplemental Information). From this test, $3.47 \times 10^{6}$ elements was chosen for case of $\theta=0^{\circ}, h=200 \mu \mathrm{m}$, which could not only keep the simulation precision but also save the CPU time. In the similar way, $3.74 \times 10^{6}$ elements for case of $\theta=0^{\circ} \& h=87.5 \mu \mathrm{m}, 3.90 \times 10^{6}$ elements for case of $\theta=90^{\circ} \& h=87.5 \mu \mathrm{m}$, and $3.45 \times 10^{6}$ elements for case of $\theta=90^{\circ} \&$ $h=200 \mu \mathrm{m}$ were selected as the optimum grid systems for further calculations.

For the convergence of the solutions, we typically perform an additional 1000 iterations to test the stability of the converged solution. It was found that the residuals did not obviously increase or decrease after an additional 1000 iterations (see Figs. S2-S5 in the Supplemental Information). We also observed the tracer contour plots at the cross section $(x=1,2$, and $3 \mathrm{~mm})$, for example, for case of $\theta=90^{\circ}$, $h=87.5 \mu \mathrm{m}$, after 1100 iteration steps, 1700 iteration steps, and 2200 iteration steps, the mass fraction distribution does not change (see Fig. S6 in the Supplemental Information). Hence, these solutions can be considered to be converged.

The no-slip boundary condition was set at the walls. We also applied the parabolic velocity profiles (defined with a user-defined function) at the circular inlet of the tube as the fully developed velocity profile (FDVP), which can be expressed in the following equation,

$V(y, z)=2 \bar{V}\left(1-\frac{(y-A)^{2}+(z-B)^{2}}{r^{2}}\right)$

where $\bar{V}$ is the mean velocity, $(A, B)$ is the coordinate of the center for each inlet, $r$ is the inner radius of inlet tube, $r=50 \mu \mathrm{m}$. (more details on the definition and setting of the FDVP, see Figs. S7 and S8 in the Supplemental Information).

Based on the simulated data, the mixing efficiency at sampled section $x=4 \mathrm{~mm}$ is calculated by the following equation:

$\sigma_{s}=\left(1-\sqrt{\sigma^{2} / \sigma_{\max }^{2}}\right) \times 100 \%$

where $\sigma=\sqrt{1 / n \sum_{n=1}^{n}\left(C_{i}-C_{\infty}\right)^{2}}$, in which $C_{i}$ (from 0 to 1 ) is the value of the mass fraction at computational cell $i(i=0 \ldots \mathrm{n}), \mathrm{C}_{\infty}$ is the mass fraction value for fully mixing. $\sigma_{\max }$ is the maximum standard deviation (no mixing at the inlet). The value of $\sigma_{\mathrm{s}}$ varies from 0 for no mixing to 1 for fully mixing.

\subsection{Simulated flow patterns in different inlet configurations}

The direction of the vortex formed relative to the surface between the fluids, can be varied by changing the parameters $h$ and $\theta$. Here in this work, the $R e$ was defined with the characteristic length the square capillary channel, which was $400 \mu \mathrm{m}$. When the fluids are introduced with $R e=120$ from the inlets into the square capillary, and $h=87.5 \mu \mathrm{m}$, (i.e., the plane which the inlet tubes are positioned is near to one side wall), the contact surface, represented by a green stripe, is slightly elongated to the other side wall at $\theta=0^{\circ}$ [Fig. 1(b)], while at $\theta=90^{\circ}$, contact surface is stretched and folded [Fig. 1(c)], because of the transverse uni-rotation of fluid elements. When $h=200 \mu \mathrm{m}$, (i.e., the inlet tubes are placed in the middle plane of the square tube), the shape of the contact surface, at $\theta=0^{\circ}$, displays no substantial change [Fig. 1(d)], whereas the stretching and folding of the contact surface in $\theta=90^{\circ}$ case, appears to be more pronounced along the $x$-axis [Fig. 1(e)], because of the transverse bilateral-rotation of fluid elements.

At a fixed $R e$ of 120 (the mean velocity at inlets is $3 \mathrm{~m} / \mathrm{s}$ ), four different flow patterns can be differentiated. By only changing the confluence angle between two fluids, their mass transport and mixing behavior were characterized. In all four cases, both inlet streams come into contact in the square channel with a sudden expansion, which will result in a sudden change of the mean velocity of the flow. The interaction between inlet streams are also different among these four conditions. At $\theta=0^{\circ}, h=87.5 \mu \mathrm{m}$, a single vortex in $z$-axis direction exists almost in one stream issuing from Inlet 2, while both fluids flow layer by layer through the square channel [Fig. 2(a)]. Single vortex in z-axis direction cannot effectively disturb the contact surface between both fluids, in which condition, the mixing mainly depends on molecular diffusion, and therefore, two fluids cannot be well mixed [Fig. 1(b) and Fig. 2(a)]. However, when the single vortex changed its direction to $x$ axis, at condition of $\theta=90^{\circ}, h=87.5 \mu \mathrm{m}$, the fluids are rotated from one side to the other, and also they are twisted with each other, which definitely enhances the area of the contact surface. This single vortex flow [Fig. 2(b)], like an invisible stirrer, is capable of breaking the laminar state, thereby increasing the advection within fluids [Fig. 1(c)]. When the inlet tubes are located in the middle plane of the square tube $(h=200 \mu \mathrm{m})$, there exists a vortex pair in $z$-axis direction $\left(\theta=0^{\circ}\right)$, but the vortex pair, evolved in the fluid from Inlet 2 , does not enlarge the contact line in transverse plane, hence, the mixing effectiveness is not improved in this case [Fig. 1(d) and Fig. 2(c)] over the one of $\theta=0^{\circ}$, $h=87.5 \mu \mathrm{m}$. When $\theta=0^{\circ}, h=200 \mu \mathrm{m}$, the direction of the vortex pair is shifted to $x$-axis. Under this condition, these two steams entwined with each other, therefore, the contact surface is enlarged toward two directions inside the square channel and also folded [Fig. 1(e) and Fig. 2(c)]. From Fig. 1 and Fig. 2, we found that although single vortex or vortex pair can be developed in the channel, the contact surface may not be effectively perturbed, only when the direction of the vortex matches the surface, the mixing potential can be enlarged.

\subsection{Pressure drops for the four geometries}

The pressure drop in the square channel for four geometries with different inflow confluence conditions as a function of the Re number is depicted in Fig. 3. We find that the pressure drops obtained for the $\theta=90^{\circ}$ cases are always higher than those for the $\theta=0^{\circ}$ cases, mainly because the vortex or vortex pair with higher strength is formed in $\theta=90^{\circ}$ cases, which results in larger flow resistance than that in $\theta=0^{\circ}$ cases. And also, this difference is becoming rather remarkable with an increase of Re. It is also noteworthy that the pressure drops in $\theta=90^{\circ}$ cases and also in $\theta=0^{\circ}$ cases share the same tendency, that is, the parameter $h$ does not evidently influence the pressure drop when $\theta$ is fixed.

\subsection{Energy dissipation related to the four geometries}

With its intrinsic non-stationarity and non-linearity, vortex motion [37] has always been a challenging topic in fluid mechanics. In this paper, we focus on the effects of strong vortices on the contact surface between fluids. The Navier-Stokes equation can be transformed into a vorticity transport equation 


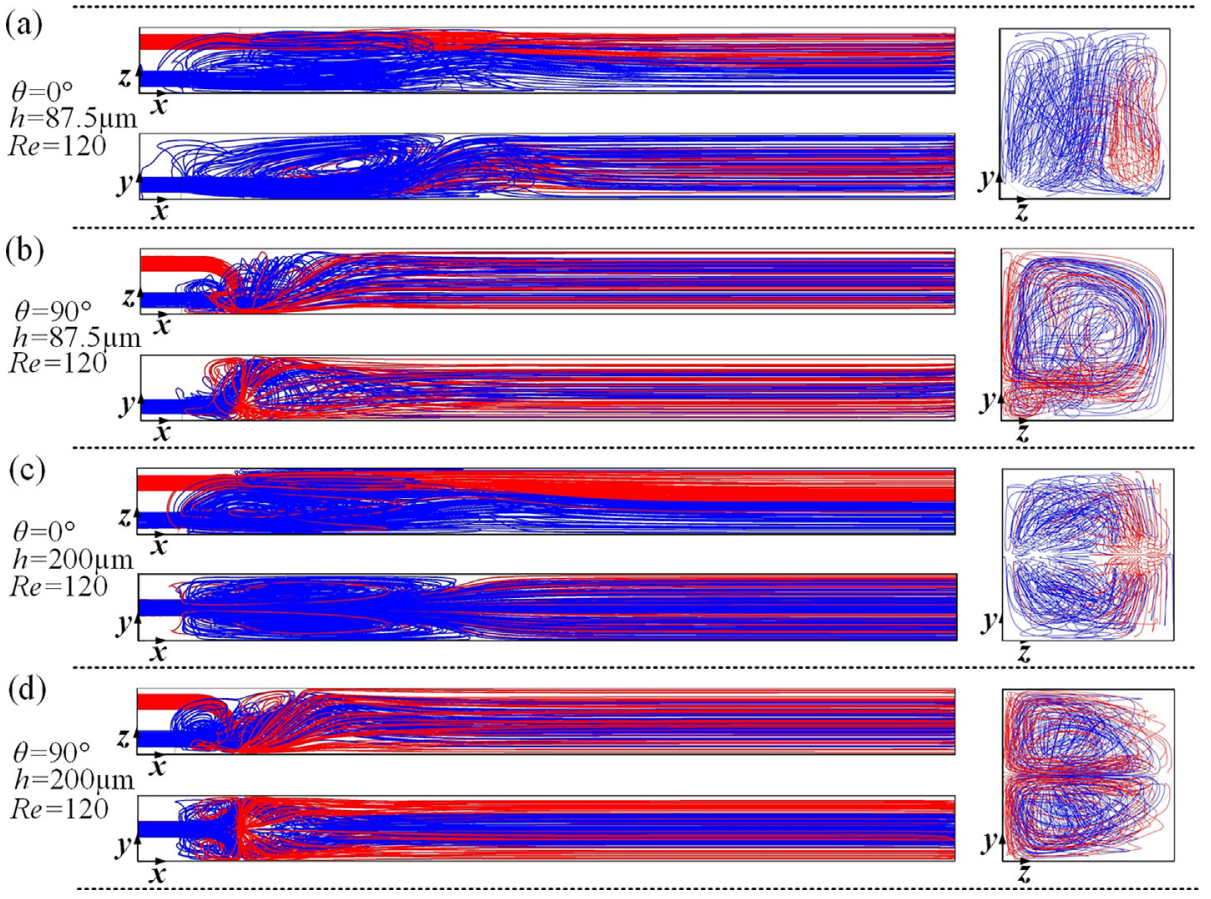

Fig. 2. The streamline patterns inside the mixing channel for each configurations observed from three directions. (a) $\theta=0^{\circ}, h=87.5 \mu \mathrm{m}$ : a single vortex produced in $z$-axis direction; (b) $\theta=90^{\circ}$, $h=87.5 \mu \mathrm{m}$ : the main fluid rotating in the $x$-axis direction; (c) $\theta=0^{\circ}, h=200 \mu \mathrm{m}$ : vortex pair developing in $z$-axis direction; and (d) $\theta=90^{\circ}$, $h=200 \mu \mathrm{m}$ : the main fluid split into two, rotating in the $x$-axis direction, i.e., a vortex pair in the $x$-axis direction under inlet conditions. Red and blue are used to distinguish between the streams entering the mixing channel from two inlets. The left insets show the interaction between two streamlines under the four inflow conditions. The distribution of streamlines in the square channel when $R e$ is 120 for each inflow from the inlets. (For interpretation of the references to color in this figure legend, the reader is referred to the web version of this article.)

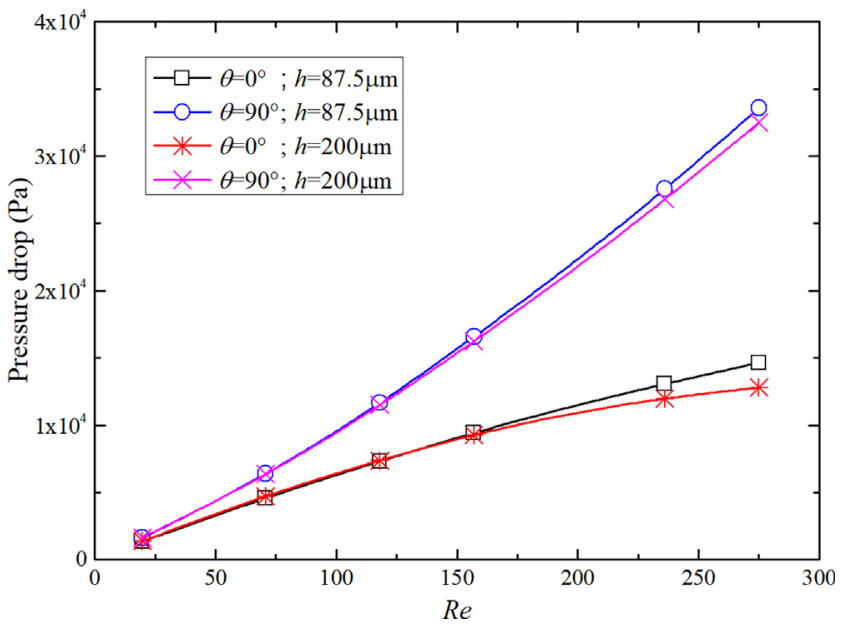

Fig. 3. Pressure drop for four geometries with different inflow confluence conditions as a function of $R e$.

$\frac{\mathrm{D} \boldsymbol{\omega}}{\mathrm{Dt}}=(\boldsymbol{\omega} \cdot \nabla) \boldsymbol{V}-\boldsymbol{\omega}(\nabla \cdot \boldsymbol{V})-\nabla\left(\frac{1}{\rho}\right) \times \nabla p+\frac{\mu}{\rho} \nabla^{2} \boldsymbol{\omega}+\nabla \times \boldsymbol{f}_{v}$

where $\boldsymbol{\omega}, \boldsymbol{V}$ are the vorticity and velocity of fluid element, and $\mu, \rho$ are the dynamic viscosity and density of the fluid, and $\nabla p, f_{v}$ are the pressure gradient and the body force acting throughout the fluid volume.

In the absence of external fields, and with gravity sufficiently negligible because of the presence of fluid in the microfluidic channels, we assume that the fluid is viscous, impressible, and barotropic, and hence the vorticity equation simplifies,

$\frac{\mathrm{D} \boldsymbol{\omega}}{\mathrm{D} t}=(\boldsymbol{\omega} \cdot \nabla) \boldsymbol{V}+v \nabla^{2} \boldsymbol{\omega}$

Vortices arise because of the fluid's viscosity and the velocity gradient; that is, the stretching and rotation of the material layers is related to the viscous dissipation. In its dimensionless form, we have

$\frac{\mathrm{D}^{*} \omega^{*}}{\mathrm{D}^{*} t^{*}}=\left(\omega^{*} \cdot \nabla^{*}\right) \boldsymbol{V}^{*}+\frac{1}{R e} \nabla^{* 2} \omega^{*}$
In flows of high $R e$, the viscous dissipation term can be safely disregarded; however, in a micro- or nano-fluidic context, this term should be taken into account, especially for situations where a vortex is present.

The conservation of energy and momentum leads to:

$\frac{\mathrm{d} E_{k}}{\mathrm{~d} t}=-2 \mu \int_{c v} \frac{1}{2} \omega^{2} d V=-\Phi$

where $E_{k}$ is the kinetic energy, and $\Phi$ the kinetic energy dissipation rate in fluid. There is a very important term,

$Q=\int_{c v} \frac{1}{2} \omega^{2} d V$

called enstrophy $[38,39]$. Here, we define the enstrophy density as

$q=\sum_{i} q_{i}=\frac{1}{2} \omega_{i} \omega_{i}$

where the enstrophy density is a scalar term that has three components. This term will provide more evidence on how vortices develop in these four physical models and which component contribute mainly to the fluid mixing and mass transport.

Fig. 4(a) shows the evolution of the mean enstrophy density along the $x$-axis direction at $R e=20$; when $\theta$ is fixed, the mean enstrophy densities show a remarkable analogous trend both in $\theta=0^{\circ}$ cases and $\theta=90^{\circ}$ cases. In situations when $\theta=0^{\circ}$, the mean enstrophy density falls to zero at around $x=1 \mathrm{~mm}$, and goes to zero for $\theta=90^{\circ}$ before $x=0.75 \mathrm{~mm}$. More importantly, when $\theta=90^{\circ}$, a peak occurs at the confluence of streams from the two inlets, which is intimately related to the quick decline of the mean velocity within a mixing length of $x=1 \mathrm{~mm}$ as the two streams flow into the outflow channel with a sudden expansion. Here we see that the kinetic energy dissipates locally more rapidly with $\theta=90^{\circ}$ than that for $\theta=0^{\circ}$, which accounts for the rapid stretching and rotating of the fluid elements in $\theta=90^{\circ}$ geometries. In terms of the three components $q_{x}, q_{y}, q_{z}$ [Fig. 4(b-d)], the biggest difference happens in the mean enstrophy density in the $x$-axis direction; the mean vorticity in the $x$-axis direction induced under $\theta=90^{\circ}$ is obviously higher than that for $\theta=0^{\circ}$. Therefore, for $\theta=90^{\circ}$, the fluid experiences a higher strength of stretching in the $y z$-plane and a rotation around the $x$-axis [Fig. 4(b)], which is also in good agreement with the occurrence of single vortex [Fig. 7(b)] or vortex pair [Fig. 7(d)] 

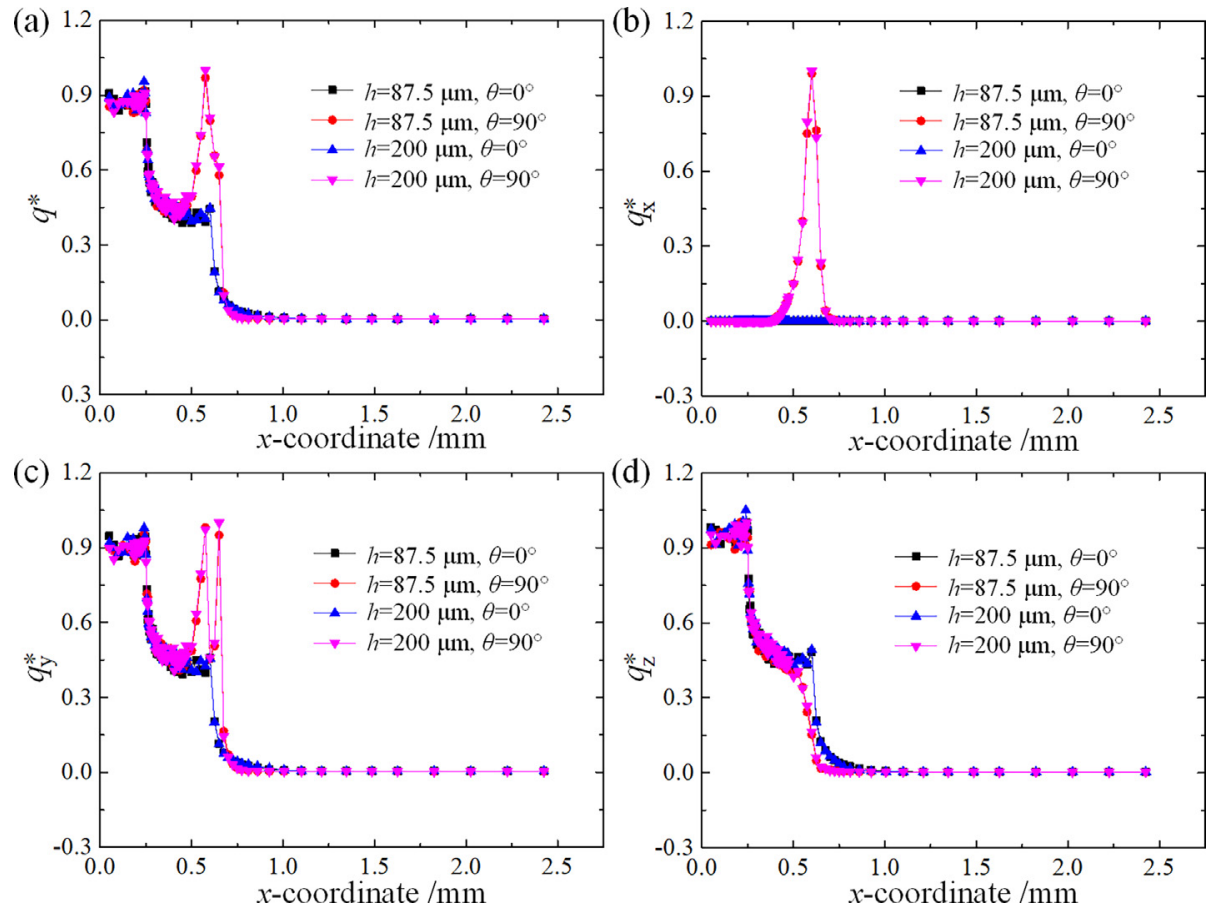

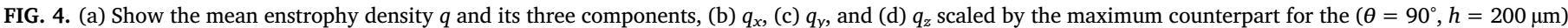
configuration on the transverse sections along the $x$-axis direction. The $R e$ for these conditions is 20 .
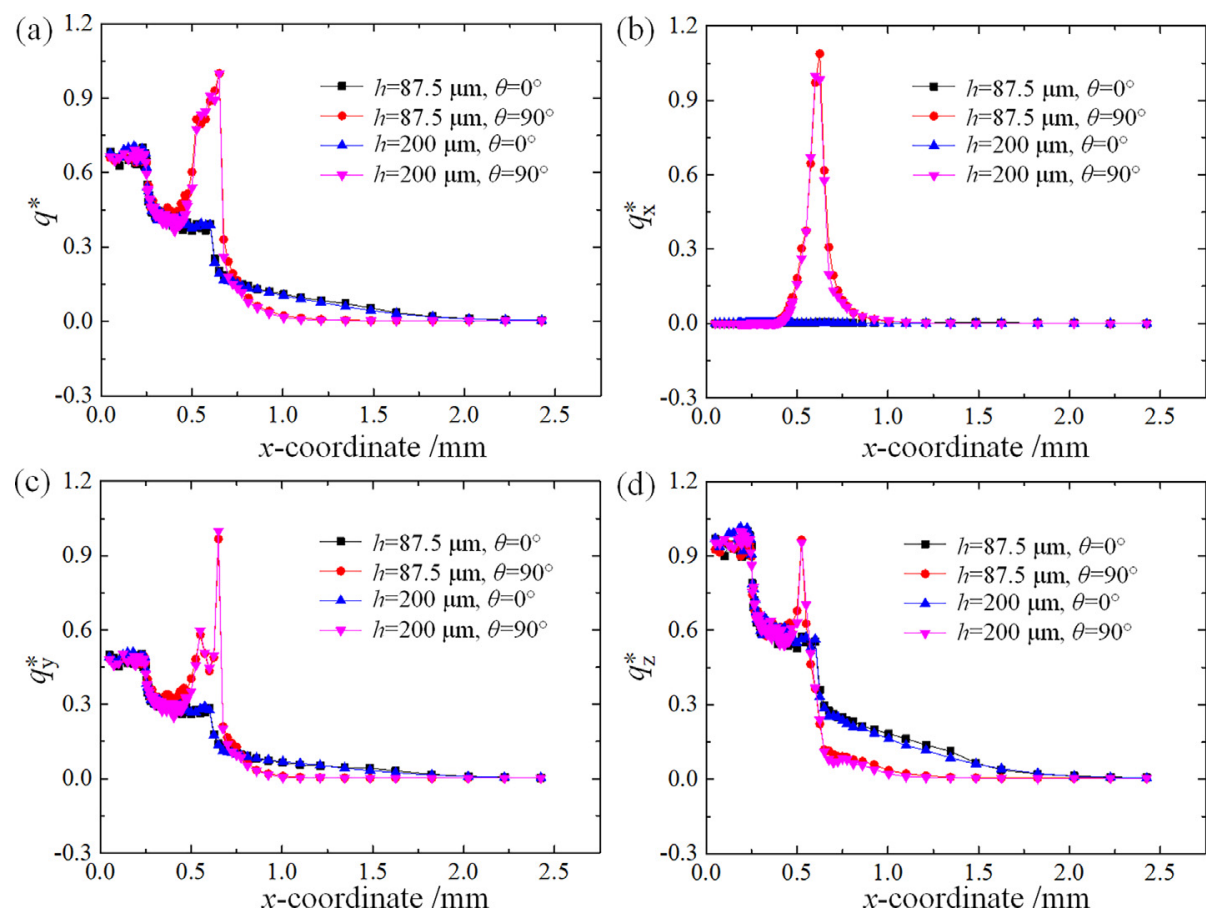

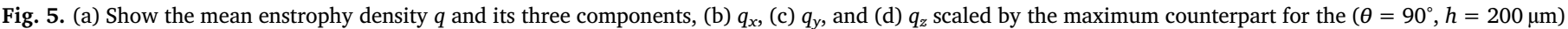
configuration on the transverse sections along the $x$-axis direction. The Re for these conditions is 120 .

with $x$-axis direction nearby the Inlet 1 . Interestingly, there are two peaks in $q_{y}$ of $\theta=90^{\circ}$ cases higher that of $\theta=0^{\circ}$ ones, which is mainly derived from stronger vortex with $y$-axis direction generated in the region between the two inlets orifices and also intense flow past the bent tube wall of Inlet 1 . Note also that $q_{z}$ for $\theta=90^{\circ}$, after a short peak, is lower than that for $\theta=0^{\circ}$ [Fig. 4(d)]. This component for $\theta=0^{\circ}$ contributes greatly to the mean enstrophy density, which is embodied in the fluid recirculation and rotation of the fluid elements around the z-axis. For further increasing Re to 120 [Fig. 5(d)], this gap between cases $\theta=0^{\circ}$ and $\theta=90^{\circ}$ is significantly enlarged because of the expansion of single vortex [Fig. 8(a)] or vortex pair [Fig. 8(c)] with $z$-axis direction.

For an increase of $R e$ to 120 , in configurations of $\theta=0^{\circ}$, the mean enstrophy density goes to zero down to $x=2 \mathrm{~mm}$, but rapidly decays to zero for $\theta=90^{\circ}$ before $x=1.25 \mathrm{~mm}$ [Fig. 5(a)]. It can be observed that the kinetic energy dissipates locally even faster with $\theta=90^{\circ}$ than that for $\theta=0^{\circ}$, that is, the flow subject to $\theta=90^{\circ}$ geometries reaches stratified state earlier that to $\theta=0^{\circ}$ geometries, but, that doesn't mean 

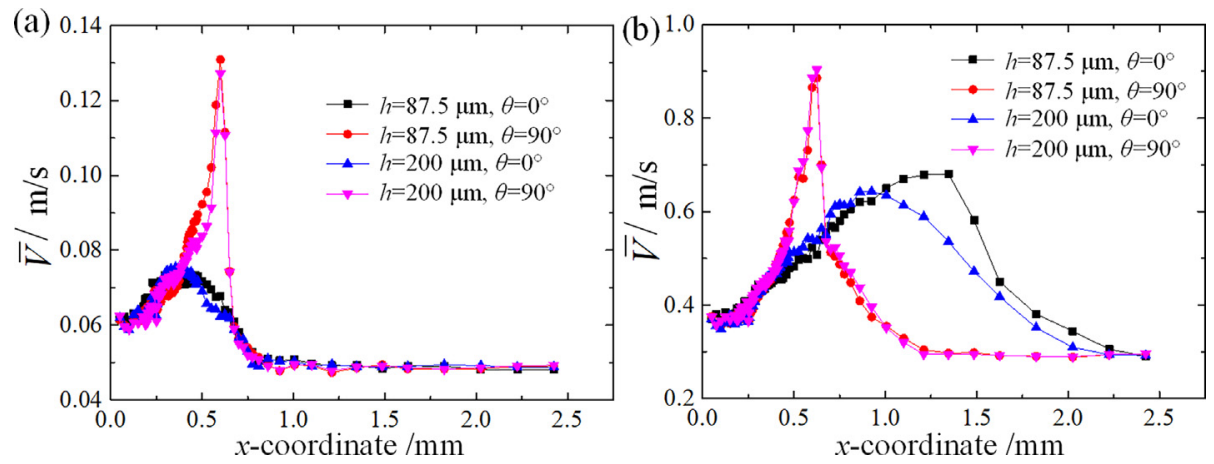

Fig. 6. The mean velocity $\bar{V}$ on the transverse sections along the $x$-axis direction. (a) $R e=20$, and (b) $R e=120$.

the flow pattern under $\theta=0^{\circ}$ is more effective than that under $\theta=90^{\circ}$. The reason will be discussed later. $q_{x}$ for these four configurations shares the same trend with that under $R e=20$. The width of the peak for $\theta=90^{\circ}$ is broadened since the vortex pair in x-axis direction acts on streams in a longer distance along the outflow channel [Fig. 5(b)]. From Fig. 5(c) and Fig. 5(d), we find that the $y$ or $z$ part of enstrophy density witnesses a slower decrease to zero for $\theta=0^{\circ}$ cases than that for $\theta=90^{\circ}$ cases, mainly because of the recirculation expanded against the outflow direction or the vortex/vrotex pair with $z$-direction pulled along the outflow direction.

\subsection{Variation of the mean velocity for the four different mixing channels}

The mean velocity along the $x$-axis direction under each combination of $(\theta, h)$ is also depicted in Fig. 6. At $R e=20$, there is a higher peak in $\theta=90^{\circ}$ cases over that in $\theta=0^{\circ}$ ones [Fig. 6(a)], which is mainly due to stronger recirculation flow generated in the region between the two inlets orifices and also intense flow past the bent tube wall of Inlet 1 , as mentioned above, and after $x=1 \mathrm{~mm}$, the flow in all cases become stable as laminar state. As Re increases to 120 [Fig. 6(b)], it will need a longer distance for the flow in $\theta=0^{\circ}$ to recover to its stratified state because the span of the vortex or vortex pair with $z$-axis direction is extended along the $x$-axis direction. The peak in $h=87.5 \mu \mathrm{m}, \theta=0^{\circ}$ is higher than that in $h=200 \mu \mathrm{m}, \theta=0^{\circ}$, which maybe result from the formation of secondary vortex following with the single vortex in the mixing channel of $h=87.5 \mu \mathrm{m}, \theta=0^{\circ}$. Because of a higher energy dissipation around the confluence region in $\theta=90^{\circ}$ cases depicted in Fig. 5(a), the flow is earlier laminarized in $\theta=90^{\circ}$ cases over the $\theta=0^{\circ}$ cases.

\subsection{The analysis on the 3-D structures in the four mixing channels identified through $\lambda_{2}$-criterion}

\subsubsection{Under $R e=20$}

The 3-D vortical structures for these channels with different inlet configurations under $R e=20$ and 120, identified from the numerical velocity field based on the $\lambda_{2}$-criterion $[30,33,40,41]$, are shown in Figs. 8 and 9 , respectively. $\lambda_{2}$ is the second largest eigenvalue of the tensor $\left(S^{2}+\Omega^{2}\right)$, where $S$ and $\Omega$ are the symmetric and antisymmetric part of the velocity gradient tensor $\nabla \boldsymbol{V}$, respectively. $\boldsymbol{S}$ is related to the strain of the fluid element, also referred to as the rate of strain tensor, and $\Omega$ is related to the rotation of the fluid element, also called vorticity tensor. A vortex core is defined as a connected region with two negative eigenvalues, i.e., $\lambda_{2}<0$. From the isosurfaces of $\lambda_{2}$ [Fig. 7], the streams flow from the inlets of circular tubes into a square tube with an expansion, producing totally separated patterns in $\theta=0^{\circ}$ cases and narrow connections in $\theta=90^{\circ}$ cases. For configuration of $\theta=0^{\circ}$, $h=87.5 \mu \mathrm{m}$, there are two bubbles originated from the inlets orifice edges as $R e=20$, tending to be tilted to central region of the square mixing channel [Fig.7(a)], compared with the case of $\theta=0^{\circ}$, $h=200 \mu \mathrm{m}$ where the bubbles exhibit a bilaterally symmetrical structures [Fig. 7(c)]. On the cutting plane $z=100 \mu \mathrm{m}$, a single vortex in $z$ direction forms near the Inlet 2 , while a vortex pair in $-z / z$-direction is generated at Inlet 2. When the situation is switched to $\theta=90^{\circ}$ geometries, the bubbles developed from the inlets tend to merge at the confluence region of the streams, and grow up toward the opposite side to the plane the inlet tubes locate [Fig. 7(b)]. However, for $\theta=90^{\circ}$, $h=200 \mu \mathrm{m}$, there are two connecting joints between the two bubbles, which is beneficial to convection between the two streams [Fig. 7(d)]. On the transverse section at $x=675 \mu \mathrm{m}$, a single vortex in $x$-direction forms adjacent to the orifice of Inlet 1 as $\theta=90^{\circ}, h=87.5 \mu \mathrm{m}$ [Fig. 7(b)], whereas a vortex pair $-x / x$-direction appear around the orifice of Inlet 1 as $\theta=90^{\circ}, h=200 \mu \mathrm{m}$ [Fig. 7(d)]. Although different vortex pattern is generated for each inflow confluence condition, the mass transport and mixing mainly depend on diffusion on the contact surface, since the vortex does not possess enough strength to rotate and fold the streams in transverse plane, especially in $\theta=0^{\circ}$ cases, where the two streams are under notable segregation.

\subsubsection{Under $\operatorname{Re}=120$}

For an increase of $R e$ to 120 , as depicted in Fig. 8, the produced vortical structures become more complex and abundant. In case of $\theta=0^{\circ}, h=87.5 \mu \mathrm{m}$ [Fig. 8(a)], the so-generated vortexes are elongated and grow up toward the center of the mixing channel, to an extent that these two blobs are connected, that is, the vortexes evolved from the two inlets orifice edges merged into one at this joint region. And also a small separate blob appears as the flow moves on, which can be rendered a secondary vortex in $z$ direction as shown in the plane of $z=100 \mu \mathrm{m}$. For configuration with $\theta=0^{\circ}, h=200 \mu \mathrm{m}$, there is one vortex pair in $z$ direction, represented by two pairs of vortical legs with a pair of weak connections at the confluence of the streams, as shown in Fig. 8(c). On the planes of $z=300$ and $100 \mu \mathrm{m}$, which respectively cut the inlet 1 and inlet 2 , two vortex pairs can be observed. On the plane of $z=200 \mu \mathrm{m}$, there is one weak vortex pair, which connects the upper and lower vortical legs at the confluence of the two streams [Fig. 8(c) and also see Fig. S9 in the Supplemental Information]. Along the flow direction, the upper two legs and lower ones are almost separated in different streams, i.e. the vortex pair does not rotating or folding the contact surface but almost stirs the fluid it locates, therefore this vortex pattern is not effective for fluid mixing. On the plane of $z=100 \mu \mathrm{m}$ which cuts the stream from the Inlet 2 , an enlarged vortex pair can be seen and the similar pattern appears on the plane of $z=300 \mu \mathrm{m}$. At $\theta=90^{\circ}, h=87.5 \mu \mathrm{m}$, the inflated recirculation bubbles on the inlet orifices interact with each other and merge at the confluence of the streams, around the plane of $x=700 \mu \mathrm{m}$, as a result, a strong vortex leg and a weak one form in $x$-direction [Fig. 8(b)]. The strong vortex can efficiently rotate the two streams since it almost spans the whole transverse plane. And the small one at the corner quickly diminishes before $x=800 \mu \mathrm{m}$. For case of $\theta=90^{\circ}, h=200 \mu \mathrm{m}$, the flow pattern is different from that under condition of $\theta=90^{\circ}, h=87.5 \mu \mathrm{m}$, two 
(a)

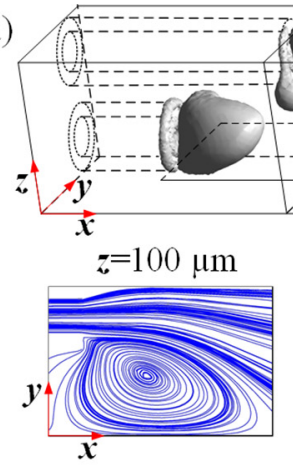

Single vortex

$\theta=0^{\circ} ; h=87.5 \mu \mathrm{m} ; R e=20$

(c)

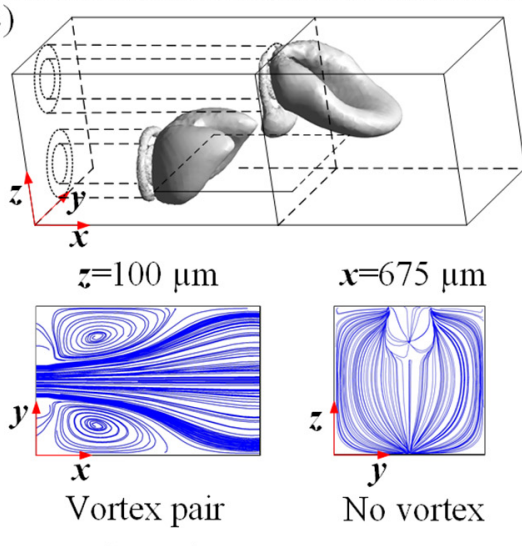

(b)
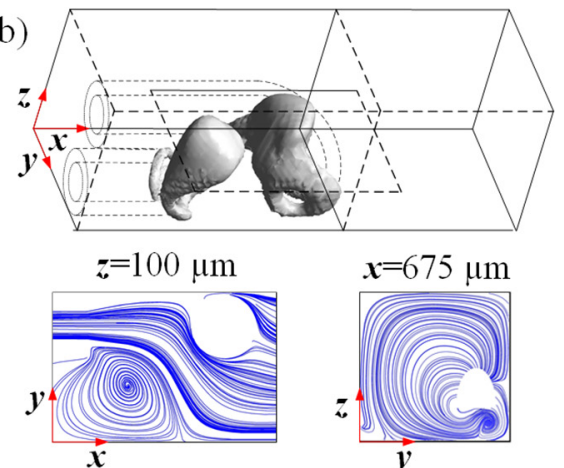

Single vortex

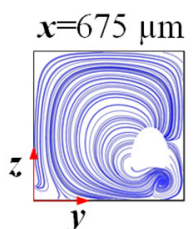

Single vortex $\theta=90^{\circ} ; h=87.5 \mu \mathrm{m}, R e=20$
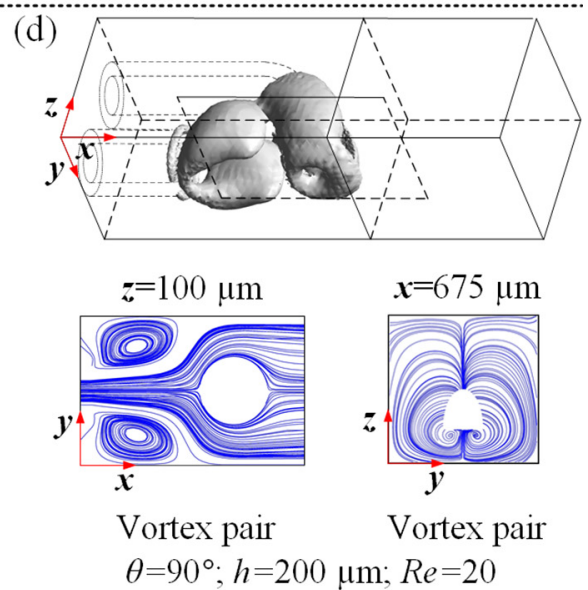

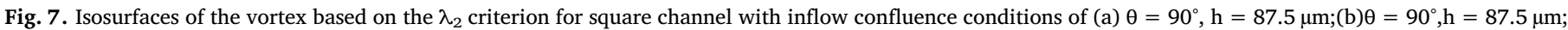
(c) $\theta=90^{\circ}, \mathrm{h}=200 \mu \mathrm{m}$; and (d) $\theta=90^{\circ}, \mathrm{h}=200 \mu \mathrm{m}$ at $\mathrm{Re}=20$.

equally strong legs are formed by counter-rotating vortices [Fig. 8(d)], which can split and rotate the streams into two rolls and certainly speed up the contact surface renewal rate, thereby resulting in a higher mixing performance on the two streams entering the square channel. The vortex pair can be observed on the selected transverse planes, with vortex cores gradually inclining to the center of the channel from $x=700 \mu \mathrm{m}$ to $x=1000 \mu \mathrm{m}$.
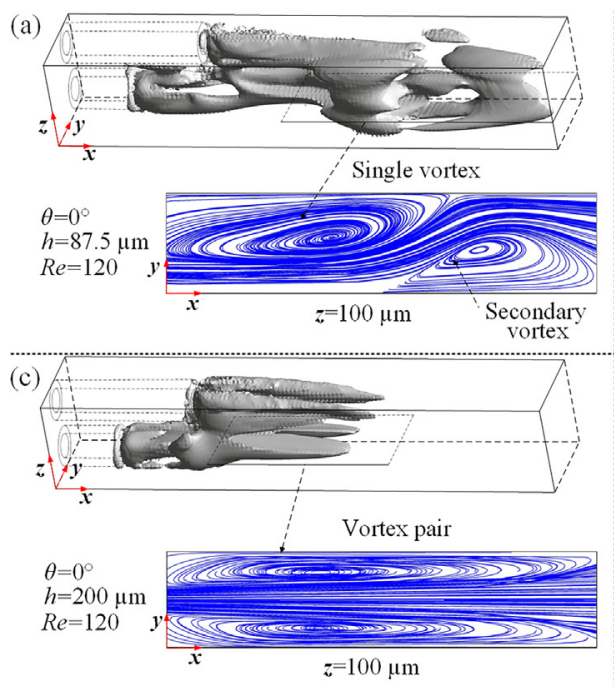

\section{Experimental validation}

\subsection{Fabrication and experimental procedure}

To inject inlet fluids into the main mixing channel (square capillary tube with $400 \mu \mathrm{m}$ in width, World Precision Instruments, Inc.), we chose cylindrical glass capillary tubes (I.D. $100 \mu \mathrm{m}$, O.D. $170 \mu \mathrm{m}$, World Precision Instruments, Inc.). Using a microforge and a microgrinder

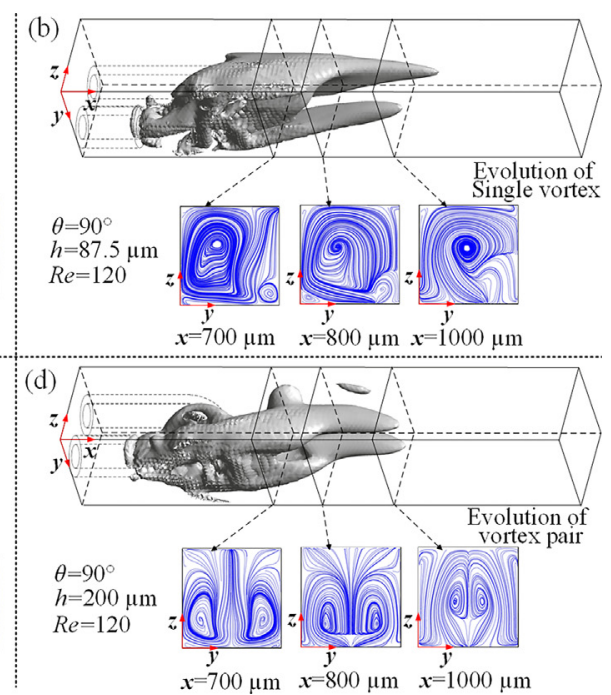

Fig. 8. Isosurfaces of the vortex based on the $\lambda_{2}$ criterion for square channel with inflow confluence conditions of (a) $\theta=0^{\circ}, \mathrm{h}=87.5 \mu \mathrm{m}$;(b) $\theta=90^{\circ}$, $\mathrm{h}=87.5 \mu \mathrm{m}$; (c) $\theta=0^{\circ}, \mathrm{h}=200 \mu \mathrm{m}$; and (d) $\theta=90^{\circ}, \mathrm{h}=200 \mu \mathrm{m}$ at $\mathrm{Re}=20$. 

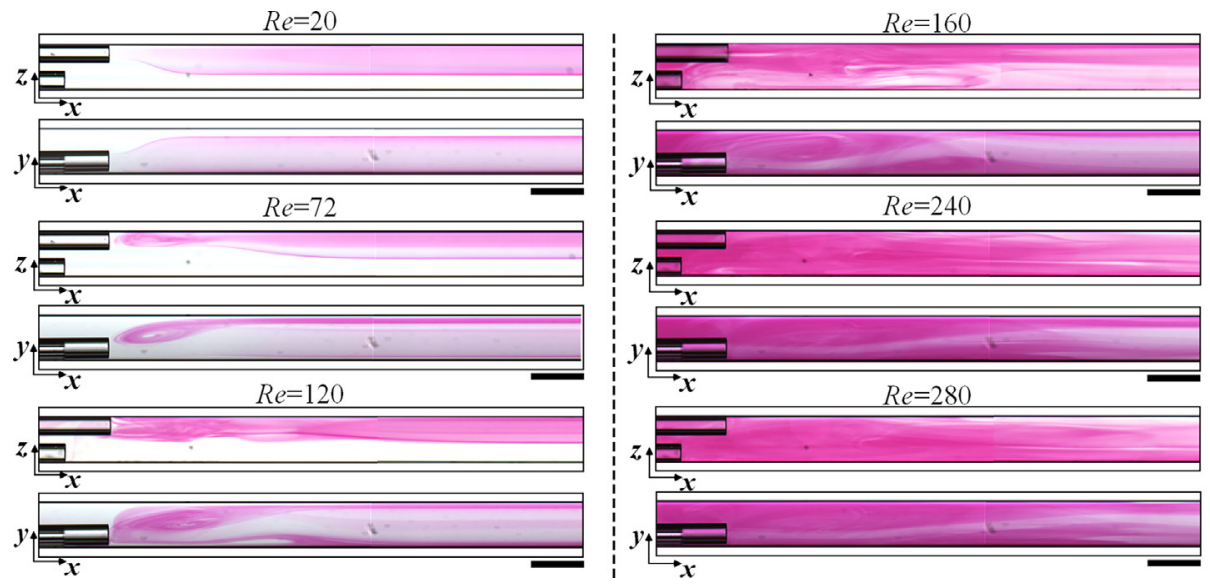

Fig. 9. Bi-direction view of mixing status obtained from experimental results with $\theta=0^{\circ}, h=87.5 \mu \mathrm{m}$, at Re varying from 20 to 280 . Each scale bar is $400 \mu \mathrm{m}$. See also Supplemental Movie 1.

(MF-900 and EG-401, Narishige, Tritech Research, Inc.), one round tube is bent to the angle $(\theta)$ of interest. Here, an angle of $90^{\circ}$ is needed, and the orifices of the tubes can be ground finely. Subsequently, these two round tubes are inserted into the square tube to form an in-out system. An accurate alignment of the tubes is sought to ensure that the tubes are located on the same plane at a distance of $h$ to the side wall of the square tube. This geometry with different combinations of $\theta$ and $h$ can lead to different flow profiles because of the interaction between fluids emanating from the inlets.

As for the working fluids used in experimental procedure, we prepared two colorless fluids that are: solution A is aqueous alkali composed of $2.4 \mathrm{~g}$ sodium hydroxide (solute A), $30 \mathrm{~g}$ DI water and $30 \mathrm{~g}$ ethyl alcohol; and solution $\mathrm{B}$ is the indicator containing $1.5 \mathrm{~g}$ phenolphthalein (solute B), $30 \mathrm{~g}$ DI water and $30 \mathrm{~g}$ ethyl alcohol. When we performed the experiment, these two fluids were injected into the mixing channels by two syringe pumps (Harvard Apparatus, PHD ULTRA). A Re range of $20 \sim 280$ was accomplished by controlling the syringe pump, and the corresponding flow rate varied from $236 \mu \mathrm{L} / \mathrm{min}$ to $3300 \mu \mathrm{L} / \mathrm{min}$. The mixing process in the microchannel was monitored under a microscope (BX53, Olympus, Japan), and the required images could be obtained using a CCD camera (RETIGA2000R, Qimaging, Canada).

\subsection{The experimental results of the four mixing channels}

The experimental results [Figs. 9-12] feature evolving flow patterns under different $R e$ ranging from 20 to 280 in microchannels with different modes of inflow confluence because of different combinations of $\theta$ and $h$. We find that a single vortex formed for conditions $\left(\theta=0^{\circ}\right.$, $h=87.5 \mu \mathrm{m}$ ) [Fig. 9, also see the Fig. S10 in the Supplemental Information and the Supplemental Movie 5] whereas vortex pair emerges [Fig. 11] as $h$ is elevated to the middle position $(h=200 \mu \mathrm{m})$, and these elliptic vortices of the $y$-axis direction are stretched to larger spans along the $x$-axis direction as Re increases to 160 . Interestingly, when $\theta=0^{\circ}, h=174 \mu \mathrm{m}$, an asymmetric co-rotating vortex pair appeared nearby the confluence of the two streams [see Fig. S11 in the Supplemental Information and the Supplemental Movie 6]. In Fig. 12, the main fluid rotates in the $x$-axis direction, whereas the contact surface is split into two strands rotating around the $x$-axis (also see Figs. 1(e) and 2(d)), whereas the fluid in the mixing channel rotates in one roll at $R e=72$. More importantly, a common characteristic of the flow patterns [Figs. 9-12] is evident in that chaos tends to be induced in flows near the inlet exit orifice under higher $R e=280$ (the $R e$ in both inlet tubes under this situation is higher, around 700). Moreover, the length for the transition from chaotic to laminar flow becomes shorter for $\theta=90^{\circ}$. From the uniformity of the red bands parallel to the $x$-axis distributed in the channel, the $\theta=90^{\circ}$ geometries are capable of stirring fluids in higher $R e$ with shorter mixing lengths. In addition, the streamlines in the channel under different conditions in the numerical calculation are found to be consistent with striations produced in each experiment (Figs. 9-12, and supplemental Videos). Note that the appearance of more and thinner striations near the outlet under higher Re reflects the higher level of mass transfer [Figs. 10 and 12].

Single vortex and vortex pair in the $z$-axis direction are observed at
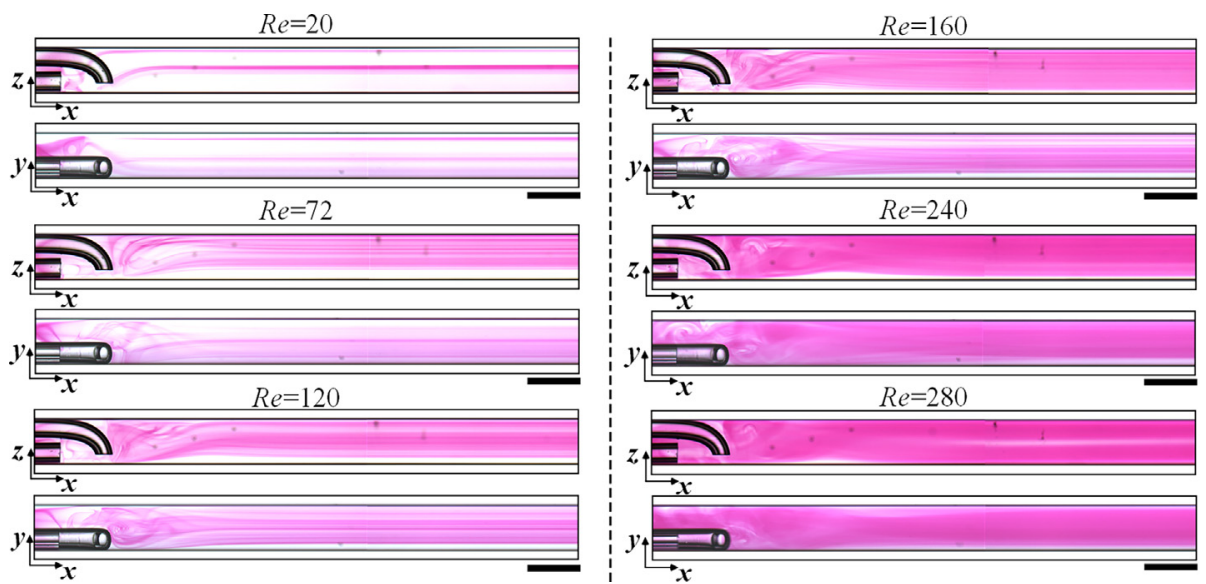

Fig. 10. Bi-direction view of mixing status obtained from experimental results with $\theta=90^{\circ}, h=87.5 \mu \mathrm{m}$, at $R e$ varying from 20 to 280 . Each scale bar is $400 \mu \mathrm{m}$. See also Supplemental Movie 2. 

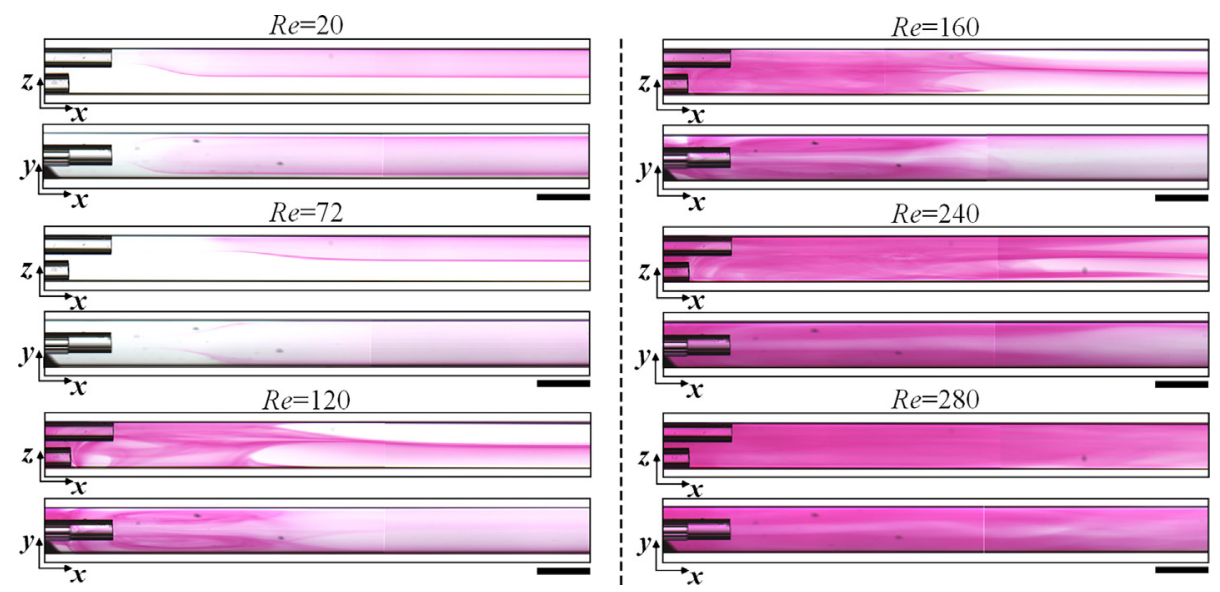

Fig. 11. Bi-direction view of mixing status obtained from experimental results with $\theta=0^{\circ}, h=200 \mu \mathrm{m}$, at Re varying from 20 to 280 . Each scale bar is $400 \mu \mathrm{m}$. See also Supplemental Movie 3.

Re of 72 and 120 in Fig. 9 and Fig. 11, respectively, and the spans of the vortices are enlarged as Re increases. In Fig. 10 and Fig. 12, fluid rolling around the $x$-axis is evident. The contact surface is being folded, spread, and rotated into numerous stripes and an even more homogeneous color is distributed in the channel at higher Re. Especially, it was found that the vortex pair evolves at the confluence of the fluids under $R e=120$ [Fig. 11], which agrees very well with the flow pattern depicted in Fig. 2 (c). Combined Fig. 12 with Fig. 2(d), it was demonstrated that the vortex pair in $x$-axis direction can significantly enhanced the mixing quality.

\subsection{Quantification of the mixing efficiency of the four channels}

The efficiency for stretching and rotating the fluid elements can be thought of as not solely the fraction of the energy being dissipated locally [42], but also the rate of enstrophy dissipating to zero. The enstrophy under $\theta=90^{\circ}$ disappears rapidly before $x=1 \mathrm{~mm}$ [Fig. 5(a)], which agrees with the state of flow where after $x=1 \mathrm{~mm}$, the fluid undergoes a transition from transverse flow with higher vorticity to laminar flow [Figs. 10 and 12]. This phenomenon is very akin to flow laminarization [43]. From disturbances in cases of $\theta=90^{\circ}$ compared with $\theta=0^{\circ}$ case, the flow in $\theta=90^{\circ}$ tends to develop more easily into turbulence with increasing $R e$ [44].

To quantify the mixing strength as a function of $R e$ (ranging from 40 to 280) at a fixed length $x=4 \mathrm{~mm}$, the mixing fraction, based on the deviation of the intensity distribution, is calculated from $\sigma_{e}=1-1 / N \sum_{i=1}^{N}\left[\left(I_{i}-I_{\min }\right) /\left(I_{\max }-I_{\min }\right)\right]^{0.5}$, where $N, I_{i}, I_{\max }$, and $I_{\min }$

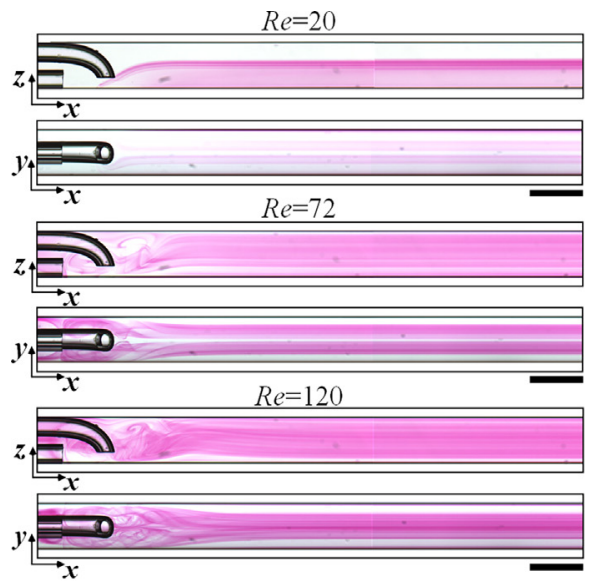

are respectively the total number of pixels, the intensity at pixel $i$, the intensity of pixels pointing to the unmixed solution, and the intensity at pixels where the solutions are fully mixed. Here $I_{\min }$ was obtained after the phenolphthalein indicator and alkali solution of equal volume were introduced into the square channel and kept overnight to guarantee they were fully mixed. Then we compared six lines of intensities with the fully mixed intensity $I_{\min }$. To obtain accurately the intensity distribution over the cross section at $x=4 \mathrm{~mm}$, we measured six lines of intensities on three equally spaced layers along the y-axis direction and another three layers in the $z$-axis direction. We focused the required planes using the above-mentioned microscope (in Section 3.1) and the corresponding images were obtained by the CCD camera; two middle layers in the $y$ and $z$ directions for each $R e$ condition are shown in Figs. 9-12. The captured images were processed using image processing software (ImageJ, NIH) and finally the mixing efficiency was quantified [Fig. 13(b)].

As the Fig. 13 shown, the trends for the simulated mixing efficiencies $\sigma_{s}$, calculated by Eq.(2) [Fig. 13(a)] are consistent with that of the experimental results [Fig. 13(b)]. And there are still some differences, for example, the overall values of the simulated results are a little bigger than that of the experiment ones.

Fig. 13 presents the fluid-mixing capabilities of four geometric configuration $(\theta, h)$. A slight increase from $20 \%$ to $45 \%$ occurs in situations $\theta=0^{\circ}$, and a substantial increase from $20 \%$ to more than $80 \%$ for $\theta=90^{\circ}$ as $R e$ increases from 40 to 280; in particular, for $\theta=90^{\circ}$, $h=200 \mu \mathrm{m}$ at $R e=280$, the averaged mixing fraction is $\sigma=91 \%$. In addition, for the $\left(\theta=90^{\circ}, h=200 \mu \mathrm{m}\right)$ configuration, the mixing

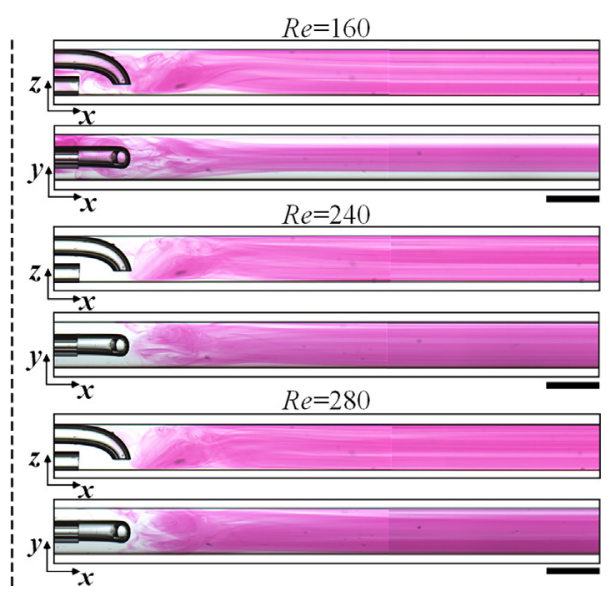

Fig. 12. Bi-direction view of mixing status obtained from experimental results with $\theta=90^{\circ}, h=200 \mu \mathrm{m}$, at $R e$ varying from 20 to 280 . Each scale bar is $400 \mu \mathrm{m}$. See also Supplemental Movie 4. 
(a)

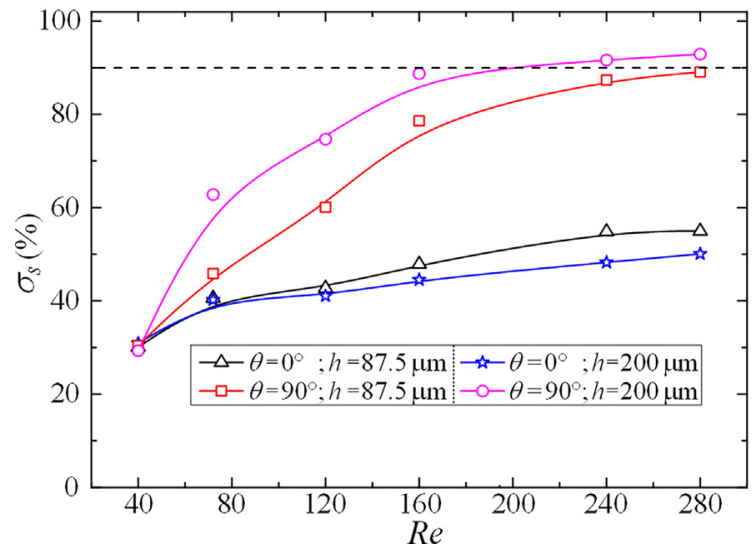

(b)

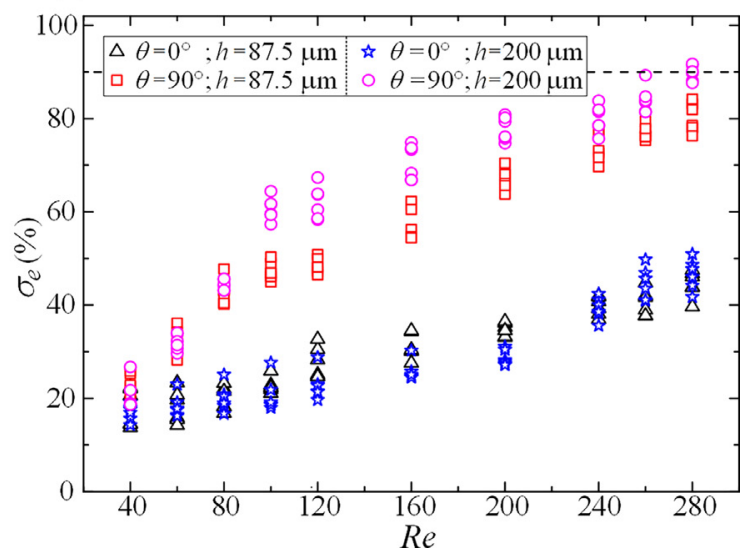

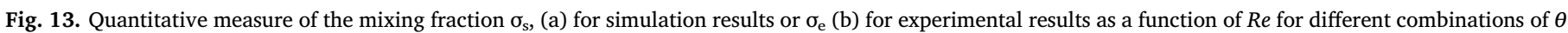

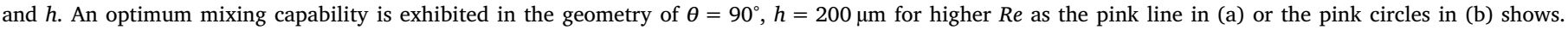

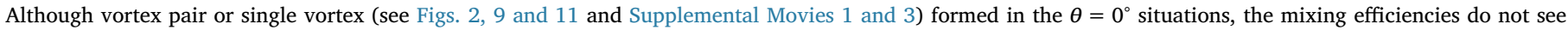

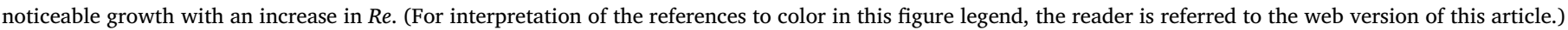

length 190 [6] for a $90 \%$ mixing fraction approaches $4 \mathrm{~mm}$. And for $\theta=0^{\circ}$ cases, $l 90$ will be longer. The mixing length $l 90$ for all cases tends to be shortened as Re increases. The reason is that the transverse stirring strength for the $\theta=90^{\circ}$ configurations is enhanced, but the mixing efficiency in $\theta=0^{\circ}$ cases is always lower than that in $\theta=90^{\circ}$ ones. To further explain the reason, from the simulation results [Fig. 7 (b \& d) and Fig. 8(b \& d)], we can see that the vortical structures are significantly enlarged when the Re increases from 20 to 120 , which mainly contributes to a higher mass renewal rate on the contact surface between two fluids [Fig. 1 (c \& e)]. And the experimental results [Figs. 10 and 12] also demonstrated that the mixing performances in $\theta=90^{\circ}$ cases were obviously enhanced as Re increased.

\section{Summary and conclusion}

Based on the numerical and experimental analysis, we assessed whether the vortex, in quickening the convection and mass transport on contact surface between fluids, depends not only directly on its strength but also strongly on the angle between it and the contact surface.

The parameter $h$ determines the symmetry of the co-rotating vortex pair; when $\theta=0^{\circ}, h=200 \mu \mathrm{m}$, the vortices are found to be symmetric and equal in size, forming a co-rotating vortex pair, which is elongated along the $x$-axis direction and tends to be unstable as $R e$ increases. When $h$ decreases to $87.5 \mu \mathrm{m}$, the vortex pair turns into a single vortex along the $z$-axis. Also, when $87.5 \mu \mathrm{m}<h<200 \mu \mathrm{m}$, an asymmetric co-rotating vortex is expected to be generated when $R e=120$ or higher. The parameter $\theta$ determined the direction of the vortex pair; when $\theta=90^{\circ}$, different from $\theta=0^{\circ}$, the rotation of the fluid around the $x$-axis strengthened, and even evolved, especially at higher $R e$, into a state of chaos near the orifice of the bending tube; after that, the fluid is "combed" into a typical laminar regime. Note that because the rotation periods are short, strong convection of the material between the mixing fluids is promoted, especially under higher Re conditions.

We only showed four combinations of $\theta$ and $h$, which were selected parameter settings chosen to generate the different flow patterns in the main capillary. Note that the distance between the orifices of the inner inlet tubes is fixed at $360 \mu \mathrm{m}$ in this study, which is also an important factor for stirring the fluid, and shortening this distance will change the mixing character of the fluids.

Although the vortex pair form in case of $h=200 \mu \mathrm{m}, \theta=0^{\circ}$ with an increase of $R e$, the mixing efficiency is not enhanced that much because the disturbance is induced not on contact surface of fluid streams, but mainly in each fluid stream. On the contrary, the $\theta=90^{\circ}$ configurations can generate strong vortex (under $h=87.5 \mu \mathrm{m}, \theta=90^{\circ}$ ) /vortex pair (under $h=200 \mu \mathrm{m}, \theta=90^{\circ}$ ) of $x$-axis direction, as Re increases, to effectively rotate and fold the surfaces of the fluid streams, thereby exhibiting a higher mixing capability. Especially for the $h=200 \mu \mathrm{m}$, $\theta=90^{\circ}$ one, the mixing efficiency reaches $91 \%$ at $R e=280$.

\section{Acknowledgements}

The authors acknowledge support from the National Natural Science Foundation of China (Grant Nos. 11672095, 11872165 and 11802078), Self-Planned Task (No. SKLRS201803B) of State Key Laboratory of Robotics and System (HIT), Opening fund of State Key Laboratory of Nonlinear Mechanics.

\section{Appendix A. Supplementary data}

Supplementary data to this article can be found online at https:// doi.org/10.1016/j.cej.2019.01.055.

\section{References}

[1] B. Wang, Y. Jia, O. Lin, A microfabrication-based approach to quantitative isothermal titration calorimetry, Biosens. Bioelectron. 78 (2016) 438-446.

[2] M.A. Schneider, T. Maeder, P. Ryser, F. Stoessel, A microreactor-based system for the study of fast exothermic reactions in liquid phase: characterization of the system, Chem. Eng. J. 101 (2004) 241-250.

[3] X. Feng, Y. Jia, H. Jiang, Q. Lin, Microfabrication-based isothermal titration calorimetry using a combined in-mixing and post-mixing titration approach, Anal. Methods 10 (2018) 4665-4670.

[4] C. Hany, H. Lebrun, C. Pradere, J. Toutain, J.-C. Batsale, Thermal analysis of chemical reaction with a continuous microfluidic calorimeter, Chem. Eng. J. 160 (2010) 814-822.

[5] R. Othman, G.T. Vladisavljević, H.C. Hemaka Bandulasena, Z.K. Nagy, Production of polymeric nanoparticles by micromixing in a co-flow microfluidic glass capillary device, Chem. Eng. J. 280 (2015) 316-329.

[6] A.D. Stroock, S.K. Dertinger, A. Ajdari, I. Mezić, H.A. Stone, G.M. Whitesides, Chaotic mixer for microchannels, Science 295 (2002) 647-651.

[7] G. Hu, D. Li, Multiscale phenomena in microfluidics and nanofluidics, Chem. Eng. Sci. 62 (2007) 3443-3454.

[8] V. Hessel, H. Löwe, F. Schönfeld, Micromixers-a review on passive and active mixing principles, Chem. Eng. Sci. 60 (2005) 2479-2501.

[9] C.-Y. Lee, W.-T. Wang, C.-C. Liu, L.-M. Fu, Passive mixers in microfluidic systems: a review, Chem. Eng. J. 288 (2016) 146-160.

[10] E. Biddiss, D. Erickson, D. Li, Heterogeneous surface charge enhanced micromixing for electrokinetic flows, Anal. Chem. 76 (2004) 3208-3213.

[11] Y. Luo, J.-Z. Luo, X.-J. Yue, Y.-J. Song, G.-W. Chu, Y. Liu, Y. Le, J.-F. Chen, Feasibility studies of micromixing and mass-transfer in an ultrasonic assisted rotating packed bed reactor, Chem. Eng. J. 331 (2018) 510-516.

[12] D. Ahmed, X. Mao, J. Shi, B.K. Juluri, T.J. Huang, A millisecond micromixer via single-bubble-based acoustic streaming, Lab Chip 9 (2009) 2738-2741.

[13] C.-Y. Chen, C.-Y. Chen, C.-Y. Lin, Y.-T. Hu, Magnetically actuated artificial cilia for optimum mixing performance in microfluidics, Lab Chip 13 (2013) 2834-2839. 
[14] L.S. Méndez-Portillo, C. Dubois, P.A. Tanguy, Free-radical polymerization of styrene using a split-and-recombination (SAR) and multilamination microreactors, Chem. Eng. J. 256 (2014) 212-221.

[15] X. Feng, Y. Ren, H. Jiang, An effective splitting-and-recombination micromixer with self-rotated contact surface for wide Reynolds number range applications, Biomicrofluidics 7 (2013) 054121.

[16] P. Hermann, J. Timmermann, M. Hoffmann, M. Schlüter, C. Hofmann, P. Löb, D. Ziegenbalg, Optimization of a split and recombine micromixer by improved exploitation of secondary flows, Chem. Eng. J. 334 (2018) 1996-2003.

[17] S. Hossain, I. Lee, S.M. Kim, K.-Y. Kim, A micromixer with two-layer serpentine crossing channels having excellent mixing performance at low Reynolds numbers, Chem. Eng. J. 327 (2017) 268-277.

[18] S. Wiggins, J.M. Ottino, Foundations of chaotic mixing, Philos. Trans. R. Soc. London A: Math. Phys. Eng. Sci. 362 (2004) 937-970.

[19] S.M. Davidson, M.B. Andersen, A. Mani, Chaotic induced-charge electro-osmosis, Phys. Rev. Lett. 112 (2014) 128302.

[20] M.A. Ansari, K.-Y. Kim, K. Anwar, S.M. Kim, Vortex micro T-mixer with non-aligned inputs, Chem. Eng. J. 181-182 (2012) 846-850.

[21] Y.C. Chung, Y.L. Hsu, C.P. Jen, M.C. Lu, Y.C. Lin, Design of passive mixers utilizing microfluidic self-circulation in the mixing chamber, Lab Chip 4 (2004) 70-77.

[22] M. Long, M.A. Sprague, A.A. Grimes, B.D. Rich, M. Khine, A simple three-dimensional vortex micromixer, Appl. Phys. Lett. 94 (2009) 133501.

[23] M.Z. Bazant, T.M. Squires, Induced-charge electrokinetic phenomena: theory and microfluidic applications, Phys. Rev. Lett. 92 (2004) 066101.

[24] Z. Wu, D. Li, Micromixing using induced-charge electrokinetic flow, Electrochim. Acta 53 (2008) 5827-5835.

[25] T. Frommelt, M. Kostur, M. Wenzel-Schäfer, P. Talkner, P. Hänggi, A. Wixforth, Microfluidic mixing via acoustically driven chaotic advection, Phys. Rev. Lett. 100 (2008) 034502.

[26] X. Chen, Y. Ren, W. Liu, X. Feng, Y. Jia, Y. Tao, H. Jiang, A simplified microfluidic device for particle separation with two consecutive steps: induced charge electroosmotic prefocusing and dielectrophoretic separation, Anal. Chem. 89 (2017) 9583-9592.

[27] H. Sun, Y. Ren, W. Liu, X. Feng, L. Hou, Y. Tao, H. Jiang, Flexible continuous particle beam switching via external-field-reconfigurable asymmetric inducedcharge electroosmosis, Anal. Chem. 90 (2018) 11376-11384.

[28] C.-H. Lin, C.-H. Tsai, L.-M. Fu, A rapid three-dimensional vortex micromixer utilizing self-rotation effects under low Reynolds number conditions, J. Micromech.
Microeng. 15 (2005) 935-943.

[29] C. Galletti, M. Roudgar, E. Brunazzi, R. Mauri, Effect of inlet conditions on the engulfment pattern in a T-shaped micro-mixer, Chem. Eng. J. 185-186 (2012) 300-313.

[30] A. Mariotti, C. Galletti, R. Mauri, M.V. Salvetti, E. Brunazzi, Steady and unsteady regimes in a T-shaped micro-mixer: synergic experimental and numerical investigation, Chem. Eng. J. 341 (2018) 414-431.

[31] A. Soleymani, E. Kolehmainen, I. Turunen, Numerical and experimental investigations of liquid mixing in T-type micromixers, Chem. Eng. J. 135 (2008) S219-S228.

[32] D. Bothe, C. Stemich, H.-J. Warnecke, Fluid mixing in a T-shaped micro-mixer, Chem. Eng. Sci. 61 (2006) 2950-2958.

[33] T. Andreussi, C. Galletti, R. Mauri, S. Camarri, M.V. Salvetti, Flow regimes in Tshaped micro-mixers, Comput. Chem. Eng. 76 (2015) 150-159.

[34] N. Aoki, T. Fukuda, N. Maeda, K. Mae, Design of confluence and bend geometry for rapid mixing in microchannels, Chem. Eng. J. 227 (2013) 198-202.

[35] J. Andrade Jr, A. Alencar, M. Almeida, J. Mendes Filho, S. Buldyrev, S. Zapperi, H. Stanley, B. Suki, Asymmetric flow in symmetric branched structures, Phys. Rev. Lett. 81 (1998) 926.

[36] C.A. Cortes-Quiroz, A. Azarbadegan, M. Zangeneh, Effect of channel aspect ratio of 3-D T-mixer on flow patterns and convective mixing for a wide range of Reynolds number, Sens. Actuators, B 239 (2017) 1153-1176.

[37] P. Saffman, G. Baker, Vortex interactions, Annu. Rev. Fluid Mech. 11 (1979) 95-121.

[38] D.K. Lilly, Numerical simulation of two-dimensional turbulence, Phys. Fluids 12 (1969) II-240-II-249.

[39] C.E. Leith, Diffusion approximation for two-dimensional turbulence, Phys. Fluids 11 (1968) 671-672.

[40] J. Jeong, F. Hussain, On the identification of a vortex, J. Fluid Mech. 285 (1995) 69-94.

[41] A. Fani, S. Camarri, M.V. Salvetti, Investigation of the steady engulfment regime in a three-dimensional T-mixer, Phys. Fluids 25 (2013) 064102.

[42] J. Ottino, Mixing, chaotic advection, and turbulence, Annu. Rev. Fluid Mech. 22 (1990) 207-254.

[43] S. He, K. He, M. Seddighi, Laminarisation of flow at low Reynolds number due to streamwise body force, J. Fluid Mech. 809 (2016) 31-71.

[44] R. Cerbus, C.-C. Liu, G. Gioia, P. Chakraborty, The laws of resistance in transitional pipe flows, Bull. Am. Phys. Soc. 62 (2017). 